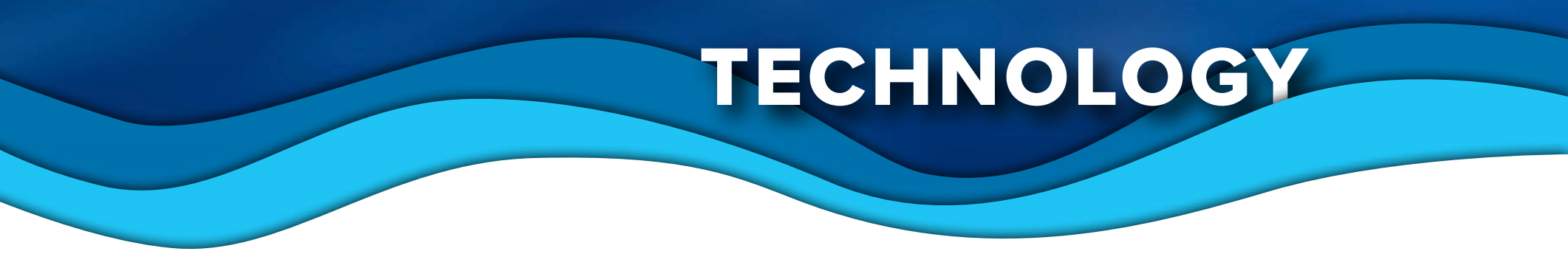

\title{
Technologies for Observing the Near Sea Surface
}

By Mariana Ribas-Ribas, Christopher J. Zappa, and Oliver Wurl

Collecting pristine observations of the sea surface from ships is challenging because research vessels destroy the integrity of the upper few meters of the ocean. This includes the sea surface microlayer (SML)-the top millimeter of the ocean-which controls the exchange of gases between the ocean and the atmosphere and plays an essential role in the dispersal of contaminants, including plastics, oil residues, and industrial organic substances. Observations of this thin surface layer at high temporal and spatial resolutions are needed to understand the ocean-atmosphere exchanges of $\mathrm{CO}_{2}$, heat, particles, and freshwater. For these reasons, new technologies for studying sea surface processes are essential to advance understanding of the ocean's health and the ocean's role in climate (Schmitt, 2018).

In situ observations of the SML are also needed to validate and calibrate surface ocean observations acquired by various sensors aboard satellites. Conventional in situ methods for obtaining sea surface measurements such as Argo floats or conductivity-temperature-depth (CTD) instruments do not take measurements of the sea surface

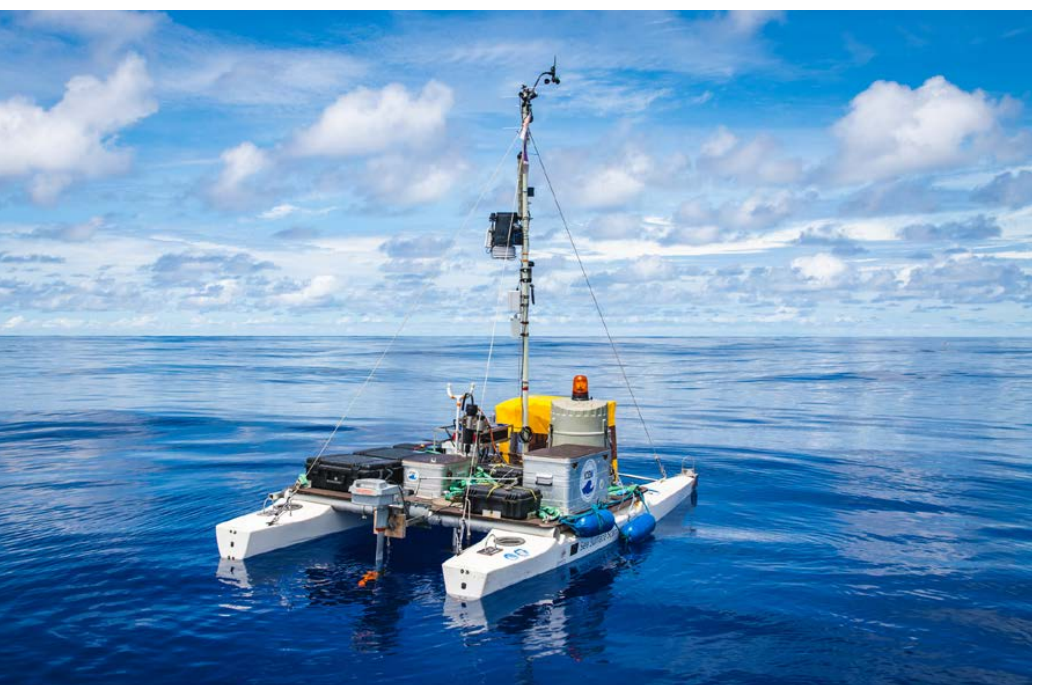

FIGURE 1. (above) The research catamaran floats peacefully in calm Fijian waters. This remotely piloted vehicle utilizes, among other things, a "skimmer" (right) - a rotating glass disk that skims the sea surface microlayer, sampling just the top $1 \mathrm{~mm}$ of the ocean. Photo credit: Alex Ingle/Schmidt Ocean Institute but instead take readings at 3-5 m depth at best. Even the size of the platforms and sensors are often too big to resolve the fine scales of observation required to understand surface ocean processes. Similar to human skin, which has different properties than the tissues and organs underneath, the ocean has a skin with different properties than the water just below. The oceanography community has traditionally used data collected from 3-5 m depth and assumed or extrapolated similar conditions occur at the surface. This would be as if a dermatologist diagnoses your skin condition by examining an X-ray.

To resolve these issues, there have been several advancements in technology and sampling methods related to the SML and near-surface layer, discussed below.

REMOTE-CONTROLLED CATAMARANS. State-of-the-art research catamarans (Figure 1) permit assessment of vertical gradients of various biogeochemical parameters either by integrating onboard sensor data or by collecting discrete water samples from the SML and from additional near-surface depths. Among other parameters, onboard sensors can record temperature, conductivity (a measure of salinity), $\mathrm{pH}$, partial pressure of $\mathrm{CO}_{2}$, and fluorescent dissolved organic matter, while dissolved inorganic carbon, total alkalinity, surface active substances, and extracellular polymeric substances are determined through analysis of discrete water samples. These catamarans also include meteorological sensor packages, and the latest version can operate autonomously, with live data transmission for mission planning. By using catamarans, we overcome the disadvantages of boats and research vessels, which break the structure of the near-surface ocean and the SML.

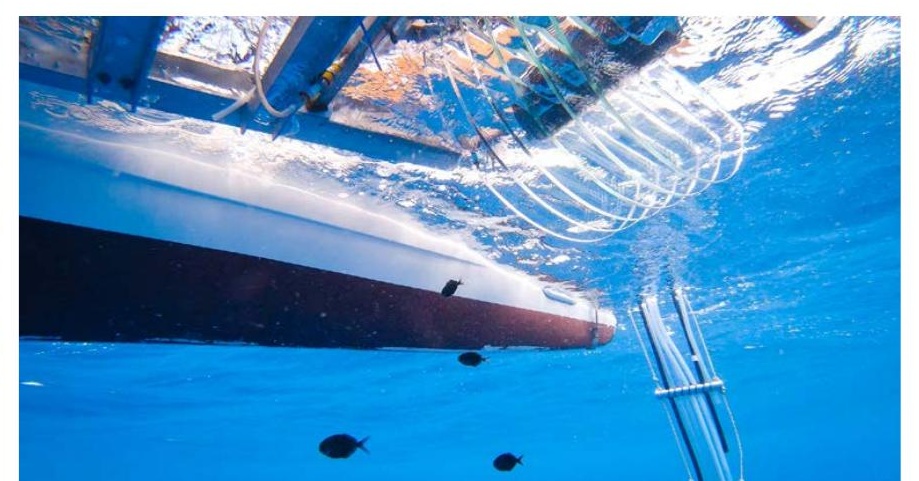




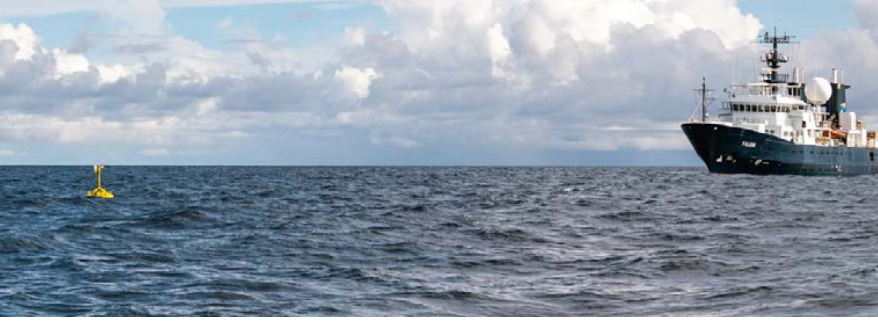

BUOYS. Drifting buoys are suitable for the study of the sea surface. Because they drift freely, any disturbance to the sea surface and its SML are minimal. We developed several drifting buoys for different scientific applications. There are drifting buoys that measure gas transfer velocities and air-sea $\mathrm{CO}_{2}$ fluxes with high spatiotemporal resolution (Sniffle). Others are equipped to incubate waters at their depths of origin, including in the SML. For example, to study the metabolic contributions of microorganisms to gas exchange processes, we use light and dark bottles on the freely drifting Surface In Situ Incubator (SISI). There are surface buoys with sensors fixed at different depths to collect data on the water's conductivity, temperature, or $\mathrm{pH}$. The Surface Processes Instrument Platform 2 (SPIP-2; Figure 2) continuously profiles temperature, salinity, current velocity, and turbulence in the top meter of the ocean up to the very surface. We have also developed an SML skimmer that will be deployed on moored data buoys along shipping lanes to monitor floating soot particles.

UNCREWED AERIAL VEHICLES. We made the first fully autonomous deployment of fixed-wing, uncrewed aerial vehicles (UAVs) on R/V Falkor cruise FK191120 in November 2019 (Figure 3). The vehicles were equipped for high endurance (12+ hours with $6.8 \mathrm{~kg}$ instrument payloads) and high data bandwidth (100+ Mbs at $50 \mathrm{~nm}$ range), and they could take-off, hover, and land vertically. These UAVs provided "eyes over the horizon," quickly covering more of the ocean than a ship and dramatically increased the research footprint available for science and discovery. Moreover, these UAVs can allow those aboard research vessels to quickly and efficiently assess targets or identify events of interest, such as cyanobacteria blooms, rather than wait for the ship to encounter a phenomenon that may not occur. After the UAV discovers a physical phenomenon of interest, the research vessel party can move to sample it while the UAV monitors its temporal and spatial evolution. These UAVs can be outfitted with remote-sensing instruments typically found aboard satellites, such as thermal cameras that provide sea surface skin temperature and hyperspectral imagers that yield ocean color data for identifying algal blooms. All of the imagery is telemetered in real time back to mission control on the ship, allowing real-time tasking and the reprogramming of data collection during the flight.
FIGURE 2. Overhead, one of the uncrewed aerial vehicles (UAVs) passes, collecting data on the sea surface below. In the water, the Surface Processes Instrument Platform (SPIP-2) collects detailed measurements of surface dynamics. Photo credit: Alex Ingle/Schmidt Ocean Institute

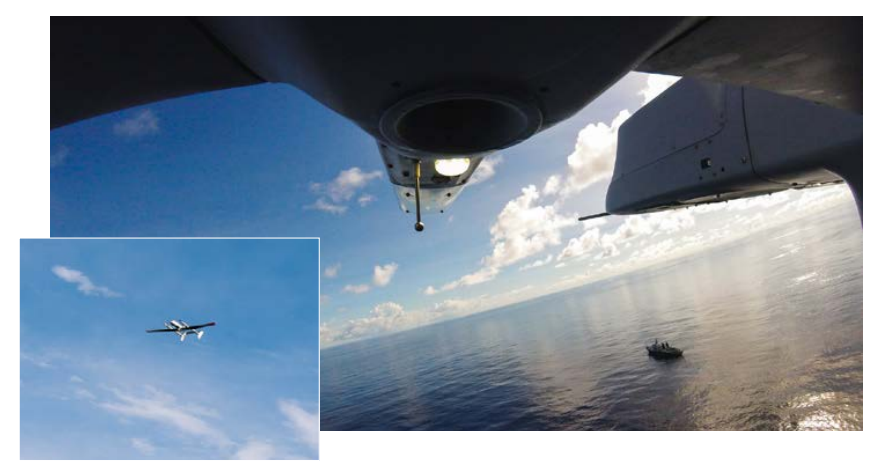

FIGURE 3. During the 2019 R/V Falkor expedition, the team tested the new capabilities of high-endurance hybrid fixed wing vertical takeoff and landing (VTOL) UAV (inset) for oceanographic applications. Pictured is the underside of one of the aircraft, making a pass of the ship. Photo credit: Alex Ingle/Schmidt Ocean Institute

\section{OUTLOOK}

While the existence of the SML has been known for many decades, we still do not have a clear understanding of how it controls air-sea interactions. It is unclear to what extent SML sampling affects the SML's integrity and the parameters of interest (especially for gases). We also expect a diurnal cycle of SML processes that we are just beginning to understand. The basic concept of the SML has also evolved. Initially, it was thought to be a separate layer that only existed during calm sea states. The SML was then shown to exist at regional to global scales and to have recurrent biofilm-like properties. Now, we are moving toward a definition of the SML as a biogeochemical reactor that exhibits vertical gradients that force the air-sea exchange of heat, energy, and mass.

Our goal is to develop readily available technology that will allow collection of data on the SML to become an integral part of research projects. Furthermore, we need to quantify SML processes and integrate them in ocean models. For example, there are still gaps in our knowledge of the ocean carbon budget. We hypothesize that we can close (or get closer to closing) the carbon budget if we introduce the SML into ocean carbon models. We cannot continue to use data collected at 3-5 m depth and extrapolate it to describe the near-surface layer because this extrapolation may introduce significant biases that affect calculations of the global carbon budget, the validation of satellite images, and our understanding of the freshwater cycle.

\section{REFERENCE}

Schmitt, R.W. 2018. The ocean's role in climate. Oceanography 31(2):32-40, https://doi.org/10.5670/oceanog.2018.225.

ARTICLE DOI: https://doi.org/10.5670/oceanog.2021.supplement.02-32 


\section{AUTHORS}

Mariana Ribas-Ribas (mariana.ribas.ribas@uol.de), Center for Marine Sensors, Institute for Chemistry and Biology of the Marine Environment, Carl von Ossietzky Universität Oldenburg, Germany. Christopher J. Zappa, Lamont-Doherty Earth Observatory, Columbia University, USA. Oliver Wurl, Center for Marine Sensors, Institute for Chemistry and Biology of the Marine Environment, Carl von Ossietzky Universität Oldenburg, Germany.

\section{ARTICLE CITATION}

Ribas-Ribas, M., C.J. Zappa, and O. Wurl. 2021. Technologies for observing the near sea surface. Pp. 88-89 in Frontiers in Ocean Observing: Documenting Ecosystems,

Understanding Environmental Changes, Forecasting Hazards. E.S. Kappel, S.K. Juniper, S. Seeyave, E. Smith, and M. Visbeck, eds, A Supplement to Oceanography 34(4), https://doi.org/10.5670/oceanog.2021.supplement.02-32.

\section{COPYRIGHT \& USAGE}

This is an open access article made available under the terms of the Creative Commons Attribution 4.0 International License (https://creativecommons.org/ licenses/by/4.0/), which permits use, sharing, adaptation, distribution, and reproduction in any medium or format as long as users cite the materials appropriately, provide a link to the Creative Commons license, and indicate the changes that were made to the original content. 